\title{
Hypotony Maculopathy After Trabeculectomy in a Patient With GAPO Syndrome
}

\author{
(D) Selim Genc, ${ }^{1}$ (1) Emre Guler, ${ }^{2}$ (b) Hanefi Cakir, ${ }^{2}$ (1) Osman Salkaci, ${ }^{3}$ (D) Fehim Esen $^{4}$ \\ ${ }^{1}$ Prof. Dr. N. Resat Belger Beyoglu Eye Training and Research Hospital, Istanbul, Turkey \\ ${ }^{2}$ Turkiye Hospital, Eye Clinic, Istanbul, Turkey \\ ${ }^{3}$ Department of Ophthalmology, Lutfi Kirdar Kartal Eye Training and Research Hospital, Istanbul, Turkey \\ ${ }^{4}$ Department of Ophthalmology, Medeniyet University Faculty of Medicine, Istanbul, Turkey
}

\begin{abstract}
This report describes a case of hypotony maculopathy developing in a patient with GAPO syndrome following a trabeculectomy with mitomycin C (MMC). A 42-year-old man with a diagnosis of GAPO syndrome underwent a trabeculectomy with an MMC application of $0.4 \mathrm{mg} / \mathrm{mL}$ for I minute. Intraocular pressure was measured at 6 to $8 \mathrm{mmHg}$ during the first weeks after the surgery. A fundus examination then revealed macular choroidal folds, retinal vascular tortuosity, and swelling in the optic nerve. At postoperative I month, additional suturing of a bleb was performed; however, the hypotony continued. Postoperative hypotony should be taken into account in patients with GAPO syndrome due to a defective fibrosis process following a trabeculectomy.

Keywords: GAPO syndrome, hypotony maculopathy, mitomycin C, trabeculectomy.
\end{abstract}

\section{Introduction}

GAPO syndrome is an autosomal recessive disease with features of growth retardation $(G)$, alopecia $(A)$, pseudoanodontia $(\mathrm{P})$, and optic atrophy $(\mathrm{O})$. Histopathological studies have demonstrated that an abnormal accumulation of extracellular material was related to defective extracellular-matrix homeostasis $(I, 2)$. The clinical presentation of the syndrome predominantly involves connective tissue (fibroblasts, chondrocytes, and osteoblasts), cardio-vascular, and ocular abnormalities.

The ophthalmological findings of the disease include progressive optic atrophy, keratoconus, and glaucoma (3-6). Hypotony maculopathy is well-known complication following a trabeculectomy, and is particularly associated with the application of antimetabolites. Presently described is a case of hypotony maculopathy in a patient with GAPO syndrome following a trabeculectomy with mitomycin C (MMC).

\section{Case Report}

A 42-year-old man with a diagnosis of GAPO syndrome was referred to our clinic due to glaucoma resistant to anti-glaucomatous medication. The physical features of the patient included a short, stocky build, a lack of scalp hair, and generalized hypotrichosis of the face. He had prominent globes and a large, slightly protruding forehead with prominent supraorbital ridges (Fig. I).

A refractive error of $+6.50-7.50 \times 180$ diopter $(D)$ was measured for the right eye and +2.50-2.75x $15 \mathrm{D}$ for the left eye. The best corrected visual acuity (BCVA) was $20 / 100$ on the right, $20 / 63$ on the left. The preoperative corneal topography showed a thinned central cornea along with increased corneal curvature. The intraocular pressure (IOP) was $32 \mathrm{mmHg}$ for the right eye and $35 \mathrm{mmHg}$ for the left eye with the medication of topical dorzolamide hydrochloride/timolol maleate and apraclonidine hydrochloride $0.5 \%$ twice a day and latanoprost at night for 10 years. A gonioscopy revealed normal iridocor- 
neal angles. The cup-to-disc ratio was $6 / 10$ for both eyes.

A trabeculectomy was performed on the right eye with MMC $0.4 \mathrm{mg} / \mathrm{mL}$ applied for I minute (Fig. 2). After lifting a limbus-based conjunctival flap, a superficial scleral flap $4 \times 4$ $\mathrm{mm}$ in size was created. Subconjunctival MMC $0.4 \mathrm{mg} / \mathrm{mL}$ was applied for I minute. Next, a 3xI-mm corneoscleral opening was made, followed by a peripheral iridectomy. Two 10-0 nylon sutures were used to close the corners of the scleral flap. Finally, the conjunctiva was sutured with 8-0 Vicryl continuous sutures (Ethicon Inc., Somerville, NJ, USA). On the first day, the IOP was $6 \mathrm{mmHg}$. During the first month following the surgery, a bleb had formed in the anterior chamber and the IOP measured between 6 and $8 \mathrm{mmHg}$. The Seidel test did not demonstrate any leakage of the bleb; however, the BCVA decreased from 20/63 preoperatively to counting fingers. A fundus examination revealed macular choroidal folds, retinal vascular tortuosity, and a swelling of the optic nerve. Postoperative optical coherence tomography demonstrated choroidal folds in the right eye (Fig. 3). At postoperative 4

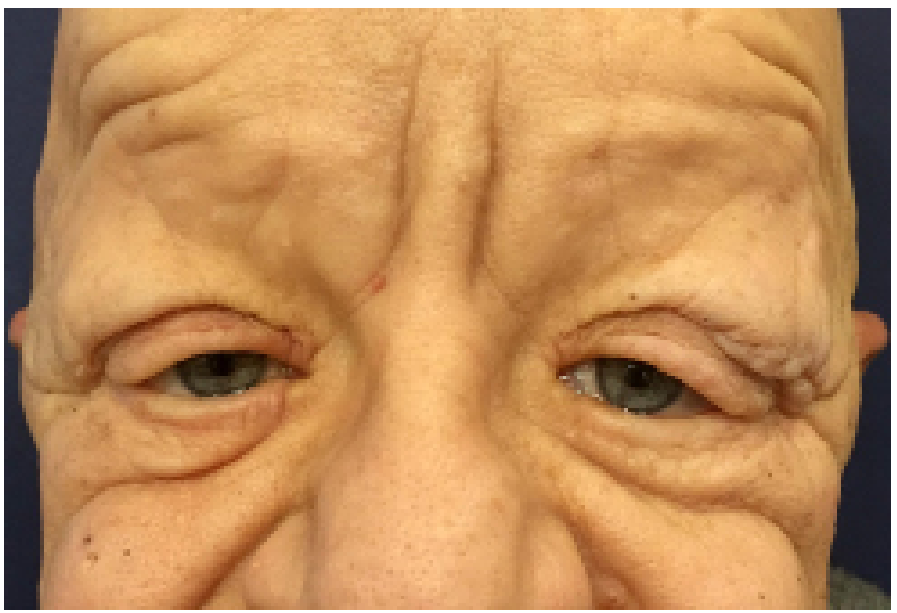

Figure I. Frontal view of the patient.

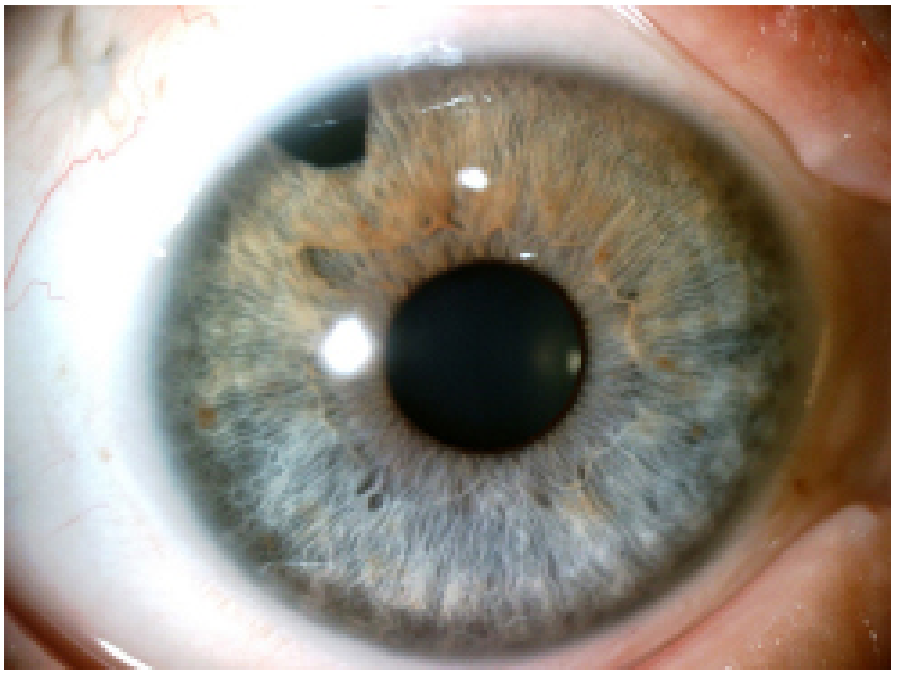

Figure 2. Right eye trabeculectomy with mitomycin C. weeks, additional suturing of the bleb was performed, but the hypotony continued.

To prevent additional hypotony, a deep sclerotomy without antimetabolites was performed for the left eye. The IOP was 8 to $1 \mathrm{I} \mathrm{mmHg} 10$ days after the operation. At postoperative 3 weeks, the IOP was 18 to $19 \mathrm{~mm} \mathrm{Hg}$. The preoperative BCVA of 20/63 decreased to 20/50. Postoperative fundus examinations did not reveal any signs of macular pathology. The histopathological evaluation of the excised scleral tissue revealed fibroblast-like cells along with extensive connective tissue (Fig. 4).
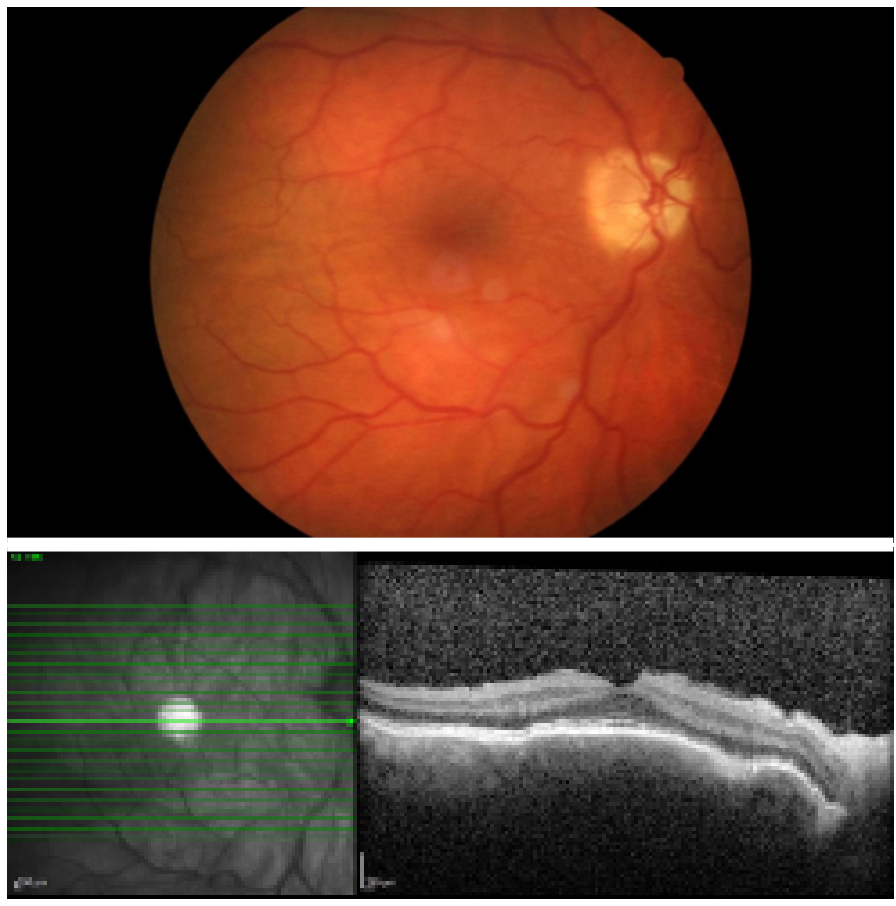

Figure 3. (a) Choroidal folds, retinal vascular tortuosity, and optic nerve swelling present in the right eye. (b) Postoperative optical coherence tomography demonstrated choroidal folds in the right eye.

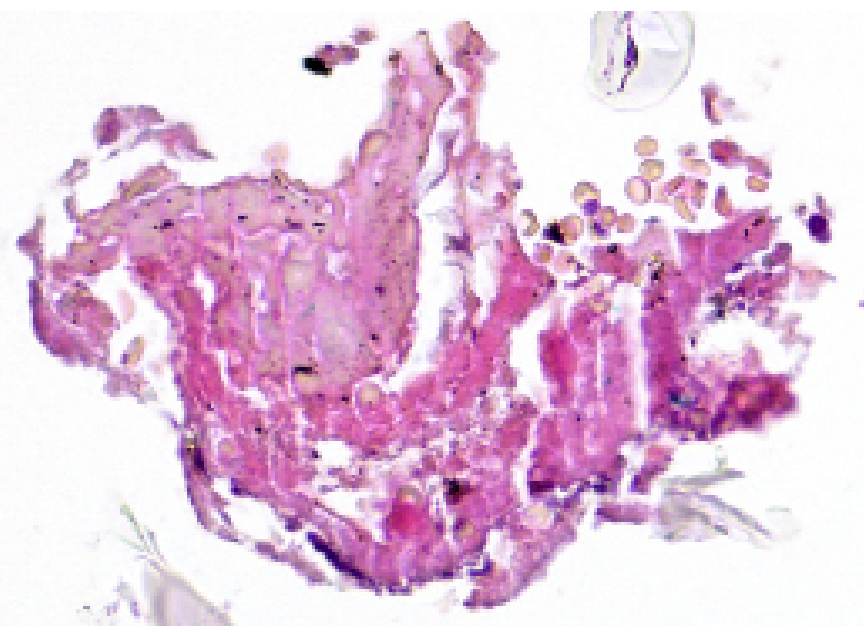

Figure 4. Histopathological evaluation of the excised scleral tissue showed fibroblast-like cells along with extensive connective tissue. 


\section{Discussion}

Growth retardation, alopecia, pseudoanodontia, and optic atrophy are the primary clinical features of GAPO syndrome. A variety of ocular findings, including ptosis, nystagmus, strabismus, myopia, megalocornea, bilateral keratoconus, band keratopathy, unilateral corneal abscess, glaucoma (including congenital), hypermature cataract, retinoschisis, retinal vein dilation, and papilledema have been reported to be related to GAPO syndrome.

GAPO syndrome is a very curious genetic disease and only a few cases have been reported in the literature. The definitive pathogenesis and the associated molecular drawbacks have not yet been identified. Degradation of extracellular matrix components has been suggested as the main defect in this syndrome, which has been reasonably associated with an enzyme deficiency connected to extracellular matrix metabolism $(I, 7)$. The main reason for the primary open-angle glaucoma may be increased resistance to aqueous humor outflow that may be associated with excessive accumulation of the extracellular connective tissue matrix and abnormally configured elastic fibrils in the trabecular meshwork.

Hypotony maculopathy generally occurs when the IOP is less than $9 \mathrm{mmHg}$. The main findings include macular choroidal folds, retinal vascular tortuosity, and optic disc edema. Hypotony maculopathy is a well-known complication of trabeculectomy, particularly related to the use of antimetabolites. Previous studies have reported that it is responsible for $14.3 \%$ of visual decline following trabeculectomy (8).

A trabeculectomy with MMC was performed in this case and hypotony occurred during follow-up. In cases with GAPO, defective extracellular matrix regulation and fibroblastic activity may cause incomplete fibrosis, which may lead hypotony after a trabeculectomy. The use of antimetabolites may also decrease fibroblastic activity in these cases. Although future studies are needed to confirm our report, postoperative hypotony should be taken into account in patients with GAPO syndrome.

\section{Disclosures}

Informed consent: Written informed consent was obtained from the patient for the publication of the case report and the accompanying images.

Peer-review: Externally peer-reviewed.

Conflict of Interest: None declared.

Authorship Contributions: Involved in design and conduct of the study (EG, SG, HÇ, OŞ, FE); preparation and review of the study (EG, SG, HÇ, OŞ, FE); data collection (EG, SG, HÇ, OŞ, FE).

\section{References}

I. Wajntal A, Koiffmann CP, Mendonça BB, Epps-Quaglia D, Sotto MN, Rati PB, et al. GAPO syndrome (McKusick 23074)--a connective tissue disorder: report on two affected sibs and on the pathologic findings in the older. Am J Med Genet 1990;37:21323. [CrossRef]

2. Meguid NA, Afifi HH, Ramzy MI, Hindawy A, Temtamy SA. GAPO syndrome: first Egyptian case with ultrastructural changes in the gingiva. Clin Genet 1997;52:1 I0-5. [CrossRef]

3. Ilker SS, Oztürk F, Kurt E, Temel M, Gül D, Sayli BS. Ophthalmic findings in GAPO syndrome. Jpn J Ophthalmol 1999;43:48-52.

4. Bacon W, Hall RK, Roset JP, Boukari A, Tenenbaum H, Walter B. GAPO syndrome: a new case of this rare syndrome and a review of the relative importance of different phenotypic features in diagnosis. J Craniofac Genet Dev Biol 1999;19:189-200.

5. Touzri RA, Goucha S, Kriaa L, Beltaif O, Fazaa B, El Andolsi $\mathrm{H}$, et al. Ocular manifestation in GAPO syndrome. Report of the first tunisian case. [Article in French]. J Fr Ophtalmol 2003;26: 1067-70.

6. Bozkurt B, Yildirim MS, Okka M, Bitirgen G. GAPO syndrome: four new patients with congenital glaucoma and myelinated retinal nerve fiber layer. Am J Med Genet A 2013;161 A:829-34.

7. Goucha S, Fazaa B, Ezzine N, Jaber K, Elandaloussi H, Abid R, et al. GAPO syndrome. [Article in French]. Ann Dermatol Venereol 2000;127:50I-4.

8. Costa VP, Smith M, Spaeth GL, Gandham S, Markovitz B. Loss of visual acuity after trabeculectomy. Ophthalmology 1993;100:599-612. [CrossRef] 